\title{
Dielectric Properties of Nanoconfined Water: A Canonical Thermopotentiostat Approach
}

\author{
F. Deißenbeck $\odot$, C. Freysoldt $\odot$, M. Todorova $\odot$, J. Neugebauer®, and S. Wippermann $\oplus^{*}$ \\ Max-Planck-Institut für Eisenforschung GmbH, Max-Planck-Straße 1, 40237Düsseldorf, Germany
}

(Received 9 March 2020; revised 14 August 2020; accepted 26 February 2021; published 2 April 2021)

\begin{abstract}
We introduce a novel approach to sample the canonical ensemble at constant temperature and applied electric potential. Our approach can be straightforwardly implemented into any density-functional theory code. Using thermopotentiostat molecular dynamics simulations allows us to compute the dielectric constant of nanoconfined water without any assumptions for the dielectric volume. Compared to the commonly used approach of calculating dielectric properties from polarization fluctuations, our thermopotentiostat technique reduces the required computational time by 2 orders of magnitude.
\end{abstract}

DOI: 10.1103/PhysRevLett.126.136803

Molecular dynamics (MD) has become an indispensable tool to efficiently simulate the behavior of a wide range of systems. Experiments, however, are often performed using basic thermodynamic variables that are different from the ones easily accessible in simulations. The need for constant temperature as opposed to the much simpler constant energy simulations is widely recognized as one of the most important examples of this kind. Consequently, significant effort has been directed at developing thermostats [1-12] with the dual purpose of (i) efficiently sampling the canonical ensemble and (ii) enabling direct control of the temperature. With the advent of robust techniques to apply electric fields in density-functional calculations [13-28], there has been continuous interest to use MD simulations to study electrically triggered processes involving electron transfer reactions, such as electrochemical reactions, field desorption, and quantum transport. For this purpose, it is necessary to conceive a potentiostat, in analogy to the theory of thermostats, in order to incorporate the electric potential as a thermodynamic degree of freedom.

The first approach of this kind was pioneered by Bonnet et al. [19]. They chose a computational setup that is grandcanonical with respect to the electronic charge, cf. Fig. 1(a). The system is then coupled to an external potentiostat, where the charge is treated as an extended spatial coordinate. Analogous to how the mechanical momenta are obtained from the derivatives of the Hamiltonian with respect to the spatial coordinates, a fictitious momentum of the charge is obtained from the derivative of the total energy with respect to the charge. This fictitious momentum is then

Published by the American Physical Society under the terms of the Creative Commons Attribution 4.0 International license. Further distribution of this work must maintain attribution to the author(s) and the published article's title, journal citation, and DOI. coupled to standard Nose-Hoover dynamics. The grandcanonical nature of their setup led Bonnet et al. to recognize that " $[\ldots]$ in its present formulation, a requirement for implementing the potentiostat scheme is the existence of an energy function $\mathcal{E}\left(r_{i}, n_{e}\right)$ that is differentiable with respect to the total electronic charge. This implies the ability to treat non-integer numbers of electrons and, in general, non-neutral systems." [19]. Unfortunately, in the context of density-functional calculations the total energy as a function of the number of electrons is a notoriously difficult quantity to compute. Furthermore, the electronic charge is a single degree of freedom. Yet, thermostating single degrees of freedom by the Nose-Hoover method requires a chain of Nose-Hoover thermostats [6]. Both requirements are serious obstacles in implementing this potentiostat concept in existing density-functional theory (DFT) codes. In fact, none of the commonly used DFT codes [29] has a potentiostat scheme that provides the opportunity to study

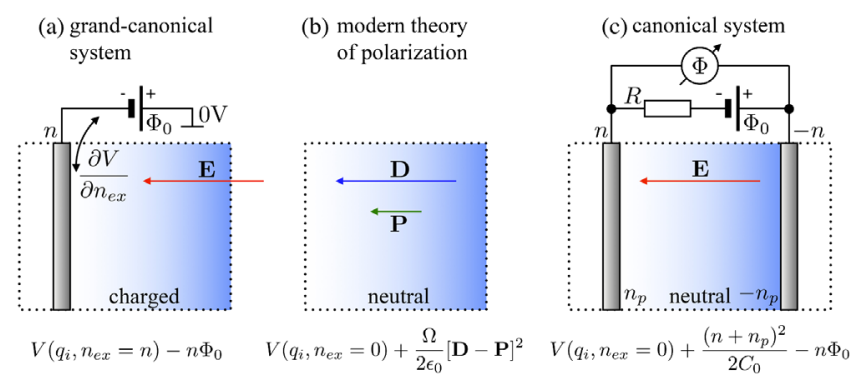

FIG. 1. Various computational setups used to include the effect of an applied electric field. (a) The simulation cell contains a single charged electrode. It is grand canonical and has an excess charge $n_{\mathrm{ex}}=n[17,19]$. (b) In the modern theory of polarization (MTP), either the electric field $\mathbf{E}$ or the displacement field $\mathbf{D}$ is used as the basic variable [16]. The simulation system is always charge neutral. (c) The simulation cell contains two electrodes with charge $n$ and $-n$. In analogy to the MTP, it is canonical and charge neutral. 
electrochemical systems with molecular dynamics and explicit water.

In the present study we show that the origin of these difficulties lies in the formally equivalent construction of the potentiostat to a thermostat: existing approaches consider a transfer of energy (thermostat) or charge (potentiostat) from the DFT cell to an external reservoir. While such a grand-canonical coupling can be straightforwardly implemented for an energy exchange, this task is severely harder for a charge transfer: either non-neutral DFT systems have to be considered or thermodynamic integration [22] between DFT systems with different total numbers of electrons. Moreover, there is also a fundamental difference between thermostats and potentiostats. For a thermostat, the system is considered to be embedded in an external temperature bath. In the physical realization of such a setup, heat transfer occurs only at the boundaries between the system and the temperature bath. Therefore, in simulations, it is common to model the simulation cell, the thermostat, and the energy exchange between them as separate entities. For a potentiostat, however, the energy exchange with the voltage source is mediated by the electric field. Since the electric field permeates the system, energy exchange occurs throughout the whole system and not just at the boundaries.

Thus, the electric field plays the same conceptual role for a potentiostat as the temperature bath for a thermostat. Yet, the electric field is an integral part of the system. In contrast to a thermostat where the bath is external, only the control mechanism of a potentiostat is external, but not the bath. We therefore propose constructing a potentiostat using the electric field as the control parameter. As will be derived in the following, this allows us to remove the need for treating charged systems. The actual implementation requires only quantities that are readily accessible in standard DFT codes, which makes it easy to integrate this potentiostat into existing electronic structure codes.

We start our derivation from the Hamiltonian given by Bonnet et al. [19] for the grand-canonical potentiostat scheme sketched in Fig. 1(a):

$$
\mathcal{H}=K\left(p_{i}\right)+V\left(q_{i}, n_{\mathrm{ex}}=n\right)-n \Phi_{0} .
$$

The explicit variables to describe atoms exposed to an external electric field are the atoms' positions $q_{i}$, their momenta $p_{i}$, and the kinetic energy of the system $K\left(p_{i}\right)$. The single electrode present in the grand-canonical setup carries a charge $n$ and is included in the simulation cell. A direct consequence of having a single charged electrode is that the system is not charge neutral but has an excess charge $n_{\mathrm{ex}}=n$. The potential energy $V\left(q_{i}, n_{\mathrm{ex}}\right)$, which describes the interatomic interaction, thus explicitly depends on the (nonzero) excess charge. We note that the potential energy $V\left(q_{i}, n_{\mathrm{ex}}=0\right)$ is simply the Born-Oppenheimer surface as computed in any DFT code.
The term $-n \Phi_{0}$ accounts for the potential of the external charge reservoir. To derive an equation of motion for the potentiostat from this Hamiltonian requires an explicit expression for the derivative of the charged system's energy $V\left(q_{i}, n_{\mathrm{ex}}\right)$ with respect to the excess charge $n_{\mathrm{ex}}$ [19].

A key concept of our proposed approach is to remove the need to compute $V\left(q_{i}, n_{\mathrm{ex}}\right)$ explicitly. As an intermediate step to achieve this aim, we consider the system shown in Fig. 1(b), which is based on concepts of the modern theory of polarization [13]. Here, the simulation cell contains the dielectric displacement field $\mathbf{D}$ created by moving a charge $n$ from the left to the right boundary. The charge itself is outside the simulation cell. The total of the left and the right charge is zero, i.e., the inner region of the cell remains charge neutral. Without loss of generality, $V$ can then be partitioned into the regular interatomic potential energy at zero excess charge $V\left(q_{i}, n_{\mathrm{ex}}=0\right)$ and the electric energy $E\left(q_{i}, n\right)$ due to the presence of the $\mathbf{D}$ field. The electric energy $E$ is given by $E=\left(\Omega / 2 \epsilon_{0}\right)[\mathbf{D}-\mathbf{P}]^{2}[16]$, where $\mathbf{P}$ is the polarization density. Thus, the difficult task of calculating $V\left(q_{i}, n_{\mathrm{ex}}=n\right)$ for the charged system is substituted by the much easier calculation of $V\left(q_{i}, n_{\mathrm{ex}}=0\right)+E\left(q_{i}, n\right)$ for a corresponding neutral system.

In a final step we extend this idea to a dielectric placed between two electrodes. The electrodes are connected to a voltage source with potential difference $\Phi_{0}$ and an internal resistance $R$, cf. Fig. 1(c). The corresponding Hamiltonian is [30]

$$
\mathcal{H}=K\left(p_{i}\right)+V\left(q_{i}, n_{\mathrm{ex}}=0\right)+\frac{\left(n+n_{p}\right)^{2}}{2 C_{0}}-n \Phi_{0} .
$$

Here, we explicitly include the two electrodes with charge $n$ and $-n$ that create the displacement field D. $C_{0}$ is the bare capacitance of the electrodes in vacuum without the dielectric and $n_{p}=\Phi / C_{0}-n$ is the polarization bound charge at the left electrode surface due to the polarization of the dielectric [36], where $\Phi$ is the instantaneous voltage measured directly across the electrodes. Note that the bound charge $n_{p}$ is an implicit function of $q_{i}$ and $n$. The Hamiltonian of the canonical potentiostat [Eq. (2)] allows us to obtain $\partial \mathcal{H} / \partial n$ analytically [30]:

$$
\frac{\partial \mathcal{H}}{\partial n}=\frac{n+n_{p}}{C_{0}}-\Phi_{0}=\Phi-\Phi_{0} .
$$

In the thermodynamic sense, the extensive charge $n$ and the intensive potential $\left(\Phi-\Phi_{0}\right)$ form a pair of conjugate variables, playing an analogous role as the volume and pressure for a barostat [37]. The reason why we are now able to obtain an analytical expression is that in the canonical system, $n$ is no longer the net charge $n_{\mathrm{ex}}$ of the system exchanged with an external bath as in the grandcanonical potentiostat approach. Rather, it is based on a charge transfer from one electrode to the other that leaves 
the total charge of the system unchanged. Hence, $\partial \mathcal{H} / \partial n$ is determined by the infinitesimal amount of energy $\partial \mathcal{H}$ required to transfer an infinitesimal amount of charge $\partial n$ between the electrodes. Thereby, Eq. (3) connects the derivative $\partial \mathcal{H} / \partial n$, which has to be computed for the microscopic quantum-mechanical system, to an external macroscopic quantity: the instantaneous voltage $\Phi$. Since our total system is charge neutral, $\Phi$ is determined directly by the total dipole moment of the charge distribution within our simulation cell. This quantity is readily accessible in DFT codes. In the following, we will use these properties of Eq. (3) to construct an equation of motion for the voltage $\Phi$ and, subsequently, for the electrode charge $n$, the control parameter of our potentiostat.

A thermostated system evolves under the influence of its internal, energy-conserving Hamiltonian and extra forces that drive the exchange of energy with the thermal bath. Similarly, a potentiostat scheme requires a forcelike term that drives the necessary changes of the electrode charge to keep the average potential constant. To obtain this force we recast Eq. (3) in differential form:

$$
\begin{gathered}
d \Phi=\frac{1}{C_{0}} d n_{p}+f d t \\
f d t=\frac{1}{C_{0}} d n
\end{gathered}
$$

The first term in Eq. (4) expresses how the potential $\Phi$ will evolve under the system's internal, energy-conserving Hamiltonian. The second term, $f d t$, is the extra forcelike term that couples to the electrode charge. This extra-force term controls on the one hand the constancy of the (average) potential. On the other hand, it has to mimic the statistical fluctuations that occur in a finite system. These two aspects are balanced by the fluctuation-dissipation theorem [38] and ensure that the system stays in the NT $\Phi$ ensemble.

Changing the potential of a capacitive system is always a dissipative process, i.e., only adapting the electrode charge to control the voltage would drain energy from the system. To avoid this energy drain, any dissipation must be accompanied by a corresponding fluctuation that returns the removed energy. In other words, the applied electric field itself must have a finite temperature: the energy dissipated by the potential control mechanism must equal on average the energy gained from thermal potential fluctuations. For the electrical circuit shown in Fig. 1(c), Johnson [39] and Nyquist [40] derived already in 1928 the relation between fluctuation and dissipation. This relation is now known as a specific case of the fluctuationdissipation theorem (FDT), and determines the variance of the potential fluctuations as well as its distribution in frequency space. Using Ohm's law and Kirchhoff's 2nd law, the current through the circuit shown in Fig. 1(c) is $d n=-\left(\Phi-\Phi_{0}\right) R^{-1} d t$. In conjunction with the FDT, we can then express the potentiostat force $f d t$ as a stochastic differential equation (SDE) [30]:

$$
f d t=\underbrace{-\frac{1}{\tau_{\Phi}}\left(\Phi-\Phi_{0}\right) d t}_{\text {dissipation }}+\underbrace{\sqrt{\frac{2}{\tau_{\Phi}} \frac{k_{B} T}{C_{0}} d W_{t}}}_{\text {fluctuation }} .
$$

$\tau_{\Phi}:=R C_{0}$ and $d W_{t}$ are the relaxation time constant of the potentiostat and a stochastic noise term given by a Wiener process, respectively. The deterministic dissipation term in Eq. (6) is equivalent to Ohm's law. The stochastic term provides the thermal fluctuations. Together, they satisfy the FDT exactly, also in frequency space [30]. Far from equilibrium, the deterministic part in Eq. (6) dominates and drives the system towards the target potential with a relaxation time of $\tau_{\Phi}$. Close to the target potential, the deterministic term becomes small and the stochastic term takes over to ensure that in thermodynamic equilibrium the canonical ensemble is correctly sampled.

The SDE Eq. (6) can be solved by employing Itō calculus [41]. Using this calculus and Eq. (5), Eq. (6) can be integrated analytically to a finite time step [30]

$$
\begin{array}{r}
n(t+\Delta t)=n(t)-C_{0}\left[\Phi(t)-\Phi_{0}\right]\left(1-e^{-\Delta t / \tau_{\Phi}}\right) \\
+N \sqrt{k_{B} T C_{0}\left(1-e^{-2 \Delta t / \tau_{\Phi}}\right),}
\end{array}
$$

where $N$ is a Gaussian random number with $\langle N\rangle=0$ and $\left\langle N^{2}\right\rangle=1$. Of course numerical integration schemes [42] are conceivable as well. Equation (7) is the central result of our derivation: All quantities entering the right side can be either directly obtained from the DFT calculation or are known from the specific setup. Thus, the electrode charge $n$ can be directly computed at each MD time step. This central result allows us to include the potentiostating process in any standard discrete time step MD scheme since the only quantity that needs to be extracted from the MD is the potential $\Phi(t)$. The scheme can be used equally well to perform $a b$ initio or empirical potential MD.

To validate our canonical thermopotentiostat scheme and to demonstrate its performance we consider a topic that had recently gained a lot of attention. Recent experiments [43] showed that a water film confined to a few nm thickness changes its dielectric behavior from the bulk dielectric constant of 80 down to 2 . Thus, the presence of solid-water interfaces appears to modify the dielectric response of water from a highly polarizable medium, which is considered to be the origin of the unique solvation behavior of water, down to a response that is close to the vacuum dielectric constant. Understanding and being able to qualitatively describe this mechanism is crucial since interfacial water is omnipresent. 
Because of the relevance of this question in fields as diverse as electrochemistry, corrosion, and electrocatalysis, several computational studies addressed dielectric properties of nanoconfined water [44-46]. These studies used either the variance of the total dipole moment fluctuations per volume, using Kirkwood-Fröhlich theory [47], or the theory of polarization fluctuations [48]. They require as an input quantity the dielectric volume to compute the dielectric constant. However, the exact location of the boundary between the electrode and the dielectric is ill defined in the presence of adsorbates, thermal motion of the electrode surface or in the context of explicit electronic structure calculations. For this reason, past studies often reported only dipole fluctuations perpendicular to the electrode surface, but not the dielectric constant $\epsilon_{\perp}$ itself [44].

Solving this problem requires explicit applied field techniques, e.g., in the spirit of Ref. [14]. Our thermopotentiostat MD allows us to address this issue directly, since our setup shown in Fig. 1(c) exactly reproduces the experimental situation. In analogy to experiment, we compute the static dielectric constant as $\epsilon_{\perp}:=\langle C\rangle_{\Phi_{0}} / C_{0}$. The calculation of the capacitances requires only the averaged charge $\langle n\rangle$ and potential $\langle\Phi\rangle$. Thus, similarly to the experiment, our approach does not require a definition of the dielectric volume.

We performed classical MD [49] of liquid TIP3P [50] water confined between two parallel electrodes. Numerical details are given in the Supplemental Material [30]; test cases are also presented in Ref. [30], and clearly demonstrate that our potentiostat alone is not only able to control the potential, but also the temperature, even in the absence of an explicit thermostat.

In Fig. 2(a), we compare our calculated static dielectric constant $\epsilon_{\perp}$ as a function of the water layer thickness to the experimental data from Ref. [43]. Red triangles and blue squares denote data points obtained from our thermopotentiostat $\mathrm{MD}$ at $\Phi_{0}=1 \mathrm{~V}$ and $\Phi_{0}=4 \mathrm{~V}$, respectively. Experimental data points measured by Fumagalli et al. [43] are shown as gray circles. Consistent with the measurements, our results display a pronounced decrease of $\epsilon_{\perp}$ compared to the static dielectric constant of bulk liquid water $\epsilon_{\text {bulk }}$ that persists for electrode separations exceeding $100 \mathrm{~nm}$. Based on this level of agreement, we therefore expect our TIP3P water model to correctly capture the impact of an interface on the dielectric properties of water.

In order to understand the origin of the decreasing $\epsilon_{\perp}$ with decreasing $d$, we computed the local static dielectric constant $\epsilon_{\perp}(z)$ as a function of the normal distance $z$ to the electrode surface [30]. Figure 2(b) shows the inverse dielectric profile $\epsilon_{\perp}^{-1}(z)$ for an electrode separation of $d=8 \mathrm{~nm}$. At the position of the electrode surface $z=0, \epsilon_{\perp}^{-1}$ drops sharply and intersects the water bulk value at $\sim 3 \AA$ above the surface. With further increasing $z$, $\epsilon_{\perp}^{-1}$ assumes negative values for interfacial water and then approaches the bulk water value in an oscillatory fashion. At a normal distance of $\sim 9 \AA$, the dielectric constant of bulk liquid water $\epsilon_{\text {bulk }}$ is recovered. The behavior in the $\sim 9 \AA$ thick interface layer reflects the well-known density modulations of water close to interfaces [51,52]; cf. Fig. 2(b) lower part.

We will now test whether the presence of the relatively thin layer of interfacial water with modified dielectric properties explains the observed huge decrease of the total static dielectric constant. Guided by the dielectric profile shown in Fig. 2(b), we partition the dielectric profile into three regions: (i) a hydrophobic gap between electrode and surface with a thickness of $d_{\text {gap }}=2 \AA$, (ii) an interfacial water region consisting of the first two water layers with a thickness of $d_{\mathrm{if}}=5.5 \AA$ and (iii) the remaining approximately bulklike region [Fig. 2(b)]. The existence of a hydrophobic gap, which is clearly visible in our data, was also suggested by Niu et al. [53] based on spectroscopic data. The effective dielectric constant of each region is obtained by integrating over the dielectric profile, yielding $\epsilon_{\text {gap }}=1.2$ and $\epsilon_{\text {if }}=17.3$ for the hydrophobic gap and interfacial water regions, respectively. We further assume that each region is approximately independent of $d$. The surrogate electrostatic model becomes then a simple plate capacitor with multiple dielectrics [inset in Fig. 2(a)]. The
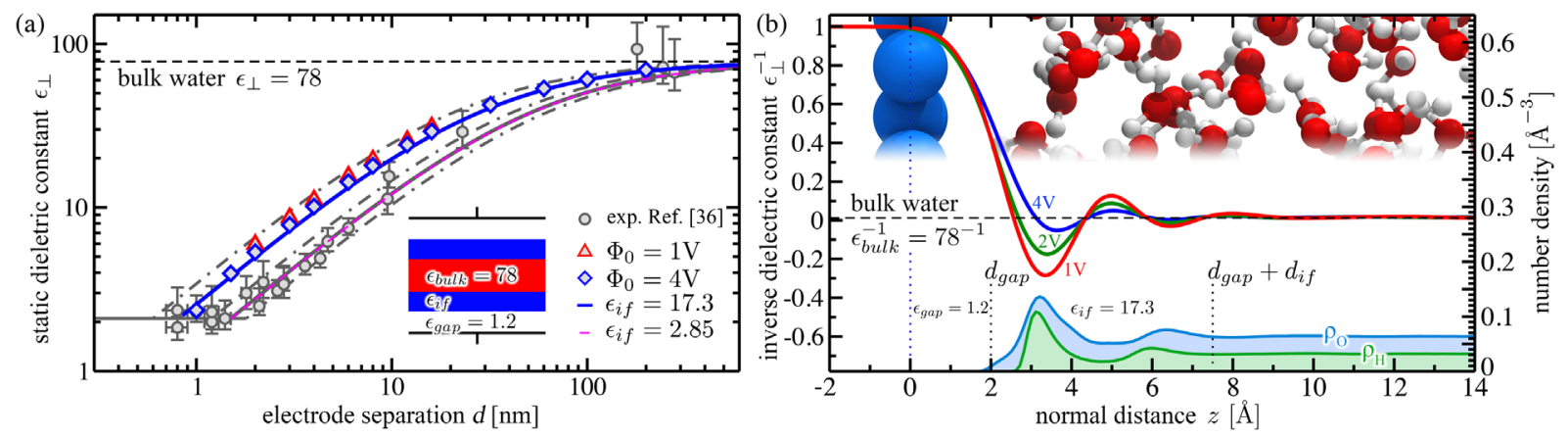

FIG. 2. (a) $\epsilon_{\perp}$ for TIP3P water as a function of electrode separation $d$, calculated using the NVT $\Phi$ ensemble. Experimental data reproduced with permission from Ref. [43]. (b) Local inverse dielectric profile and $\mathrm{O} / \mathrm{H}$ number density profiles for $d=8 \mathrm{~nm}$. The number density was computed at $\Phi_{0}=2 \mathrm{~V}$. The blue dotted line marks the position of the electrode. 
total dielectric constant is given by the analytical expression $\epsilon_{\perp}(d)=d /\left[2 d_{\text {gap }} / \epsilon_{\text {gap }}+2 d_{i f} / \epsilon_{\text {if }}+\left[d-2\left(d_{\text {gap }}+d_{\text {if }}\right)\right] / \epsilon_{\text {bulk }}\right]$. The solid blue line in Fig. 2(a) denotes $\epsilon_{\perp}(d)$ obtained by the surrogate model. Although here $\epsilon_{\perp}(d)$ was obtained from a single explicit calculation for an electrode separation of $d=8 \mathrm{~nm}$, the solid blue line accurately reproduces all other data points obtained from explicit thermopotentiostat MD simulations at different separations $d$. These findings confirm that the local dielectric properties of water close to the interface are indeed responsible for the observed reduction of $\epsilon_{\perp}$ compared to $\epsilon_{\text {bulk }}$.

The calculation of dielectric profiles from polarization fluctuations [48] requires hundreds of nanoseconds of statistical sampling, in practice enforcing the use of classical MD. In contrast, the stochastic canonical sampling of our thermopotentiostat MD in conjunction with finite electric field techniques turns out to be extremely efficient: our expression for $\epsilon_{\perp}$ [30] allows us to rely purely on thermodynamic averages rather than variances. Thus, the dielectric profiles shown in Fig. 2(b) converged within less than $4 \mathrm{~ns}$, reducing the required computational time by more than 2 orders of magnitude.

In conclusion, we devised a novel thermopotentiostat approach to sample the canonical ensemble at constant temperature and applied electric potential. Our approach (i) avoids the need to treat non-neutral simulation cells, (ii) requires only quantities that can be directly obtained from density-functional theory simulations and (iii) is straightforward to implement in any standard $a b$ initio molecular dynamics package. To demonstrate the performance of our approach we computed the thicknessdependent dielectric properties of nanoconfined water. We showed that the presence of interfaces strongly modifies the dielectric constant of an interfacial water region. This region is spatially highly confined with a thickness of only $\sim 1 \mathrm{~nm}$ (roughly two water layers). This thin region with modified dielectric properties is shown to fully capture the experimentally observed anisotropic dielectric response, persisting for distances exceeding $100 \mathrm{~nm}$. The computational efficiency of our approach is improved by more than 2 orders of magnitude compared to previous ones. In conjunction with ab initio MD, we expect our thermopotentiostat to open the door towards accurate and efficient simulations of equilibrium properties, such as interfacial dielectric constants, as well as processes such as electron transfer and electrochemical reactions.

We thank L. Fumagalli for providing the raw experimental data from Ref. [43] and D. Marx for discussions. F. D. and S.W. are supported by the German Federal Ministry of Education and Research (BMBF) within the NanoMatFutur programme, Grant No. 13N12972. Funded by the Deutsche Forschungsgemeinschaft (DFG, German Science Foundation) under Germany's Excellence Strategy-EXC 2033-Project No. 390677874 and within the framework of SFB 1394, Project No. 409476157.
Supercomputer time provided by the National Energy Research Scientific Computing Center (NERSC) Berkeley, Project No. 35687, is gratefully acknowledged.

*wippermann@mpie.de

[1] L. Woodcock, Chem. Phys. Lett. 10, 257 (1971).

[2] H. Andersen, J. Chem. Phys. 72, 2384 (1980).

[3] H. J. C. Berendsen, J. P. M. Postma, W. F. van Gunsteren, A. DiNola, and J. R. Haak, J. Chem. Phys. 81, 3684 (1984).

[4] S. Nosé, J. Chem. Phys. 81, 511 (1984).

[5] W. G. Hoover, Phys. Rev. A 31, 1695 (1985).

[6] G. Martyna, M. Klein, and M. Tuckerman, J. Chem. Phys. 97, 2635 (1992).

[7] T. Schneider and E. Stoll, Phys. Rev. B 17, 1302 (1978).

[8] M. Allen and D. Tildesley, Computer Simulation of Liquids (Oxford University Press, NY, 1991).

[9] C. P. Lowe, Europhys. Lett. 47, 145 (1999).

[10] G. Bussi, D. Donadio, and M. Parinello, J. Chem. Phys. 126, 014101 (2007).

[11] G. Bussi and M. Parinello, Comput. Phys. Commun. 179, 26 (2008).

[12] E. Braun, S. Moosavi, and B. Smit, J. Chem. Theory Comput. 14, 5262 (2018).

[13] R. Resta, Rev. Mod. Phys. 66, 899 (1994).

[14] P. Umari and A. Pasquarello, Phys. Rev. Lett. 89, 157602 (2002).

[15] M. Stengel and N. A. Spaldin, Phys. Rev. B 75, 205121 (2007)

[16] M. Stengel, N. Spaldin, and D. Vanderbilt, Nat. Phys. 5, 304 (2009).

[17] A. Lozovoi, A. Alavi, J. Kohanoff, and R. Lynden-Bell, J. Chem. Phys. 115, 1661 (2001).

[18] I. Tavernelli, R. Vuilleumier, and M. Sprik, Phys. Rev. Lett. 88, 213002 (2002).

[19] N. Bonnet, T. Morishita, O. Sugino, and M. Otani, Phys. Rev. Lett. 109, 266101 (2012).

[20] M. Otani and O. Sugino, Phys. Rev. B 73, 115407 (2006).

[21] I. Hamada, M. Otani, O. Sugino, and Y. Morikawa, Phys. Rev. B 80, 165411 (2009).

[22] J. Frenzel, B. Meyer, and D. Marx, J. Chem. Theory Comput. 13, 3455 (2017).

[23] C. Zhang and M. Sprik, Phys. Rev. B 94, 245309 (2016).

[24] T. Sayer, M. Sprik, and C. Zhang, J. Chem. Phys. 150, 041716 (2019).

[25] Q. Wan, L. Yang, M. Pander, S. Tecklenburg, A. Erbe, F. Gygi, G. Galli, and S. Wippermann (to be published).

[26] M. Ashton, A. Mishra, J. Neugebauer, and C. Freysoldt, Phys. Rev. Lett. 124, 176801 (2020).

[27] S. Surendralal, M. Todorova, M. Finnis, and J. Neugebauer, Phys. Rev. Lett. 120, 246801 (2018).

[28] O. Magnussen and A. Groß, J. Am. Chem. Soc. 141, 4777 (2019).

[29] https://dft.sandia.gov/Quest/DFT_codes.html.

[30] See Supplemental Material at http://link.aps.org/ supplemental/10.1103/PhysRevLett.126.136803 for information on the mathematical background, which contains Refs. [31-35]. In addition, an example thermopotentiostat implementation is provided, based on the internal scripting language of the LAMMPS code. 
[31] D. Gillespie, Am. J. Phys. 64, 225 (1996).

[32] D. T. Gillespie, Phys. Rev. E 54, 2084 (1996).

[33] R. W. Hockney and J. W. Eastwood, Computer Simulations Using Particles (Adam Hilger, NY, 1989).

[34] J.-P. Ryckaert, G. Ciccotti, and H. J. C. Berendsen, J. Comput. Phys. 23, 327 (1977).

[35] H. Andersen, J. Comput. Phys. 52, 24 (1983).

[36] L. Landau and E. Lifshitz, Electrodynamics of Continuous Media (Pergamon Press, Oxford, 1984).

[37] G. Bussi, T. Zykova-Timan, and M. Parinello, J. Chem. Phys. 130, 074101 (2009).

[38] H. Callen and T. Welton, Phys. Rev. 83, 34 (1951).

[39] J. Johnson, Phys. Rev. 32, 97 (1928).

[40] H. Nyquist, Phys. Rev. 32, 110 (1928).

[41] C. Gardiner, Stochastic Methods (Springer, Berlin, 2009).

[42] B. Leimkuhler and C. Matthews, Appl. Math. Res. Express 2013, 34 (2012).

[43] L. Fumagalli et al., Science 360, 1339 (2018).
[44] C. Zhang, F. Gygi, and G. Galli, J. Phys. Chem. Lett. 4, 2477 (2013).

[45] P. Loche, A. Wolde-Kidan, A. Schlaich, D. Bonthuis, and R. Netz, Phys. Rev. Lett. 123, 049601 (2019).

[46] S. Ruiz-Barragan, D. Munoz-Santiburcio, S. Körning, and D. Marx, Phys. Chem. Chem. Phys. 22, 10833 (2020).

[47] M. Sharma, R. Resta, and R. Car, Phys. Rev. Lett. 98, 247401 (2007).

[48] H. Stern and S. Feller, J. Chem. Phys. 118, 3401 (2003).

[49] S. Plimpton, J. Comput. Phys. 117, 1 (1995).

[50] W. L. Jorgensen, J. Chandrasekhar, J. D. Madura, R. W. Impey, and M. L. Klein, J. Chem. Phys. 79, 926 (1983).

[51] P. Bopp, A. Kornyshev, and G. Sutmann, Phys. Rev. Lett. 76, 1280 (1996).

[52] D. Cherepanov, Phys. Rev. Lett. 93, 266104 (2004).

[53] F. Niu, R. Schulz, A. C. Medina, R. Schmid, and A. Erbe, Phys. Chem. Chem. Phys. 19, 13585 (2017). 